\title{
MAPT Study: A Multidomain Approach for Preventing Alzheimer's Disease: Design and Baseline Data
}

\author{
B. Vellas ${ }^{1,2,3}$, I. Carrie ${ }^{1}$, S. Gillette-Guyonnet ${ }^{1,2,3}$, J. Touchon ${ }^{4}$, T. Dantoine ${ }^{5}$, J.F. Dartigues ${ }^{6}$, M.N. Cuffi, \\ S. Bordes ${ }^{8}$, Y. Gasnier ${ }^{8}$, P. Robert ${ }^{9}$, L. Bories ${ }^{10}$, O. Rouaud ${ }^{11}$, F. Desclaux ${ }^{12}$, K. Sudres ${ }^{13}$, M. Bonnefoy ${ }^{14}$, \\ A. Pesce ${ }^{15}$, C. Dufouil ${ }^{16}$, S. Lehericy ${ }^{17}$, M. Chupin ${ }^{18}$, J.F. Mangin ${ }^{19}$, P. Payoux ${ }^{20,21}$, D. Adel2 ${ }^{20}$, P. Legrand ${ }^{22}$, \\ D. Catheline ${ }^{22}$, C. Kanony ${ }^{23}$, M. Zaim ${ }^{23}$, L. Molinier ${ }^{2,3,24}$, N. Costa ${ }^{2,3,24}$, J. Delrieu ${ }^{1}$, T. Voisin ${ }^{1,2,3}$, C. Faisant ${ }^{1}$, \\ F. Lala ${ }^{1}$, F. Nourhashemi ${ }^{1,2,3}$, Y. Rolland ${ }^{1,2,3}$, G. Abellan Van Kan ${ }^{1,2,3}$, C. Dupuy ${ }^{1,2}$, C. Cantet ${ }^{1,2,3}$, P. Cestac ${ }^{1,2,3}$, \\ S. Belleville ${ }^{25}$, S. Willis ${ }^{26}$, M. Cesari, ${ }^{1,2,3}$, M.W. Weiner ${ }^{27}$, M.E. Soto ${ }^{1,2,3}$, P.J. Ousset ${ }^{1,2,3}$, S. Andrieu ${ }^{1,2,3,28}$
}

1. Gérontopôle, Department of Geriatrics, CHU Toulouse, Purpan University Hospital, Toulouse, France; 2. INSERM UMR 1027, Toulouse, France; 3 . University of Toulouse III, Toulouse, France; 4. Department of Neurology, Memory Research Resource Center for Alzheimer's Disease, University Hospital of Montpellier, Montpellier, France; 5. Geriatrics Department, Memory Research Resource Center, University Hospital of Limoges, Limoges, France; 6. INSERM U897, Memory Research Resource Center for Alzheimer's Disease, University Hospital of Bordeaux, Bordeaux, France; 7. Geriatrics Department, Hospital of Castres, Castres, France; 8. Geriatrics Department, Hospital of Tarbes, Tarbes, France; 9. Memory Research Resource Center, University Hospital of Nice, Nice, France; 10. Geriatrics Department, Hospital of Foix, Foix, France; 11. Memory Research Resource Center, Neurology Department, University Hospital of Dijon, Dijon, France; 12. Geriatrics Department, Hospital of Lavaur, Lavaur, France; 13. Geriatrics Department, Hospital of Montauban, Montauban, France; 14. Geriatrics Department, Centre Hospitalier Lyon-Sud, Lyon, France; 15. Geriatrics Department, Hospital of Princess Grace, Monaco; 16. INSERM Center U897, CIC-EC7, Bordeaux University, Department of Public Health of CHU Bordeaux, Bordeaux, France; 17. Institut du Cerveau et de la Moelle épinière, Centre de NeuroImagerie de Recherche (CENIR), InstiCRICM, UPMC/Inserm U975, CNRS 7225, Hôpital Pitié Salpétrière, Paris, France; 18. Inserm U1127, CNRS UMR 7225, Sorbonne Universités, UPMC Univ Paris 06 UMR S 1127, Institut du Cerveau et de la Moelle épinière, ICM, Inria Paris-Rocquencourt, F-75013, Paris, France; 19. CATI, NeuroSpin, CEA-Saclay Center, Gif-sur-Yvette, France; 20. INSERM UMR 825, Toulouse, France; 21. Department of Nuclear Medicine, CHU Toulouse, Purpan University Hospital, Toulouse, France; 22. Nutrition Department, Agrocampus-INRA, Rennes, France; 23. Institut de Recherche Pierre Fabre, Toulouse, France; 24. Department of Medical Information, CHU Toulouse, Toulouse, France; 25. Research Center, Institut Universitaire de Gériatrie de Montréal, Montréal, Canada; 26. Department of Psychiatry and Behavioral Sciences, University of Washington, Washington, USA; 27. University of California, San Francisco, California, United States; 28. Department of Epidemiology and Public Health, CHU Toulouse, Toulouse, France

Corresponding Author:Pr Bruno Vellas, Gérontopôle, 170 Avenue de Casselardit, TSA 40031, 31059 Toulouse Cedex 9, France - E-mail: vellas.b@chu-toulouse.fr

\begin{abstract}
OBJECTIVE: The Multidomain Alzheimer Preventive Trial (MAPT study) was designed to assess the efficacy of isolated supplementation with omega-3 fatty acid, an isolated multidomain intervention (consisting of nutritional counseling, physical exercise, cognitive stimulation) or a combination of the two interventions on the change of cognitive functions in frail subjects aged 70 years and older for a period of 3 years. Ancillary neuroimaging studies were additionally implemented to evaluate the impact of interventions on cerebral metabolism (FDG PET scans) and atrophy rate (MRIs), as well as brain amyloïd deposit (AV45 PET scans).

DESIGN, PATIENTS: 1680 subjects (mean age: 75.3 years; female: $64.8 \%$ ), enrolled by 13 memory clinics, were randomized into one of the following four groups: omega-3 supplementation alone, multidomain intervention alone, omega-3 plus multidomain intervention, or placebo. Participants underwent cognitive, functional and biological assessments at M6, M12, M24 and M36 visits. The primary endpoint is a change of memory function at 3 years, as assessed by the Free and Cued Selective Reminding test. All participants will be followed for 2 additional years after the 3-years intervention (MAPT PLUS extension study).

INTERVENTIONS: 1/ Omega-3 supplementation: two soft capsules daily as a single dose, containing a total of $400 \mathrm{mg}$ docosahexaenoic acid (DHA), i.e., $800 \mathrm{mg}$ docosahexaenoic acid per day, for 3 years. 2/ Multidomain intervention: collective training sessions conducted in small groups (6-8 participants) in twelve 120-minute sessions over the first 2 months (two sessions a week for the first month, and one session a week the second month) then a 60-minute session per month in the following three areas: nutrition, physical activity, and cognition
\end{abstract}

until the end of the 3 years. In addition to the collective sessions, individualized preventive outpatient visits exploring possible risk factors for cognitive decline are performed at baseline, M12 and M24.

BASELINE POPULATION: For cognition, the mean MMSE at baseline was 28.1 ( \pm 1.6$)$. About $58 \%$ and $42 \%$ of participants had a CDR score equal to 0 and 0.5 , respectively. Regarding mobility status, $200(11.9 \%)$ had a 4-m gait speed lower or equal to $0.8 \mathrm{~m} / \mathrm{s}$. According to the Fried criteria, $673(42.1 \%)$ participants were considered pre frail, and $51(3.2 \%)$ frail. The red blood cell DHA content was $26.1 \pm 8.1 \mu \mathrm{g} / \mathrm{g}$. Five hundred and three participants underwent baseline MRI. AV45 PET scans were performed in 271 individuals and preliminary results showed that $38.0 \%$ had a cortical SUVR $>1.17$, which gave an indication of significant brain amyloïd deposit.

DISCUSSION: The MAPT trial is presently the first largest and longest multidomain preventive trial relevant to cognitive decline in older adults with subjective memory complaints. The multidomain intervention designed for the MAPT trial is likely to be easily implemented within the general population.

Key words: Multidomain intervention, omega-3 fatty acids, prevention, Alzheimer's disease.

\section{Introduction}

M ultiple factors interact in older people with Alzheimer's disease (AD). Current evidence, based on epidemiological studies and randomized clinical controlled trials, suggests that 
nutrition, physical exercise, cognitive activity and social stimulation may improve cognitive health (1). An increase in omega-3 polyunsaturated fatty acid intake has been proposed as a possible intervention for preventing or delaying age-related cognitive decline, particularly docosahexaenoic acid (DHA) $(2,3)$. Given that prevention is the best way to decrease the burden of AD (4), it seems logical to initiate multidomain interventions addressing the multiple factors involved $(5,6)$. The Multidomain Alzheimer Preventive Trial (MAPT) study is a large and long-term trial specifically designed to test whether a multidomain intervention consisting of nutritional counseling, physical exercise and cognitive stimulation, in combination with omega-3 fatty acid supplementation, is effective in slowing cognitive decline in frail older adults at risk of cognitive decline (7). The hypothesis tested in this study is that a multidomain intervention is likely to be more beneficial than a single intervention, due to a possible synergistic effect of the tested components. This approach has another advantage: the interventions tested are widely available to millions of older adults. In addition to testing its overall efficacy, the trial includes ancillary studies to investigate brain beta-amyloïd $(\mathrm{A} \beta)$ load as well as the potential impact of interventions (multidomain intervention and/or omega-3 supplementation) on cerebral metabolism and brain atrophy rate. The cost-effectiveness of these interventions will also be assessed.

In the present paper, we describe the design and baseline data from the MAPT trial.

\section{Methodology}

The MAPT study is a phase III, multi-centered $(n=13)$, randomised, placebo- controlled trial, using a 4-arm design with 3 treatment groups (omega-3 alone, multidomain intervention alone, omega-3 plus multidomain intervention) and a placebo group. The study protocols have been approved by the French Ethical Committee located in Toulouse (CPP SOOM II) at the date of December 6, 2007 and authorized by French health authority (Ministry of Health) on December 31, 2007. Written consent was obtained from all participants. The protocol is registered on a public-access clinical trial database (www.clinicaltrials.gov) [NCT00672685)].

\section{Target Population}

The target population for MAPT includes adults aged 70 years and older, living in the community and at risk of cognitive decline. On the basis of available evidence, three main inclusion criteria were adopted for recruiting individuals:

1) Spontaneous memory complaint expressed to the general practitioner,

2) Limitation in one instrumental activity of daily living (IADL, i.e., ability to use the telephone, shop, prepare meals, do housekeeping, do one's laundry, use transportation, follow a medication schedule, or manage money ),

3) Slow walking speed (i.e., lower than $0.8 \mathrm{~m} / \mathrm{s}$, which is more than 5 seconds to walk 4 meters).

Demented subjects (8) were excluded as well as subjects with a Mini Mental State Examination (MMSE) score lower than 24 (9), dependency for the basic activities of daily living (ADL score lower than 6 [range : 0-6]) (10), and with any disease that could compromise the subject's participation. In addition, subjects who had taken omega-3 supplementation within the past 6 months were not included.

Previous studies have shown that subjective memory complaint motivating medical consultation represents a significant risk factor for dementia incident in older persons (1). Recently, studies have also observed hippocampal atrophy in subjects with memory complaints compared to those with no memory complaint (11). Recent data from several research groups have also provided evidence that self-perceived decline in cognitive performance in elderly people, even those with normal performance on cognitive tests, increases the risk of future dementia and $\mathrm{AD}$, and thus may suggest the presence of preclinical AD (12). At the Alzheimer's Association International Conference (AAIC) in July 2013, several research teams suggested targeting individuals with subjective memory complaints for preventive trials (13). We previously used this strategy to select subjects for the preventive GuidAge trial (14). Using such easy-toassess conditions may facilitate the possible translation of trial results into clinical practice. In many epidemiological studies, limitations in IADLs have also been linked to an increased risk of developing dementia $(15,16)$; as well as slow gait speed $(17,18,19)$.

\section{Outcome Measures}

The primary outcome measure in MAPT is a change in memory function after a 3 years follow up, assessed by a validated French version (20) of the Free and Cued Selective Reminding test (FCRST; (21)). This measure evaluates immediate and delayed free and cued recall of 16 words, a learning procedure used to predict AD disease progression and possible dementia (22). Progress in memory decline (reported through changes in the cognitive tests slope), rather than conversion to dementia, was recently recommended as a primary outcome for primary prevention trials (23) and recently considered by the FDA / EMEA.

Several secondary outcomes assessed efficacy of interventions on other cognitive performances, as measured by the Controlled Oral Word Association Test [COWAT] and the Category Naming Test [CNT] (lexical and categorical fluency, respectively) (24), the Digit Symbol Substitution Subtest of the Wechsler Adult 
Intelligence Scale-Revised (attention and executive function) (25), the Trail Making Test (motor activity and selective attention) (26), the MMSE (9), and the Clinical Dementia Rating Scale [CDR] (27). Two visual-analogue scales are also administered to assess memory function and the consequences of memory impairment in everyday life (28).

Other secondary outcomes assessed efficacy of interventions on:

- Physical function as measured by the Alzheimer's Disease Cooperative Study-Activities of Daily Living Prevention Instrument [ADCS-ADL PI] (dependency) (29) and the Short Physical Performance Battery [SPPB] (30) (physical performance).

- Frailty syndrome determined by using the classification system proposed by Fried et al $(31,32)$, based on assessments of grip strength, timed walking, involuntary weight loss, fatigue and physical activity.

- Depression as measured by the Geriatric Depression Scale - 15 items [GDS-15] (33).

- Tolerability to the omega-3 supplementation determined by counting the number of capsules remaining every 6 months.

- Adherence to the multidomain intervention by counting the number of sessions followed by each participant,

- And, finally, we assessed the cost effectiveness of interventions based on the Incremental CostEffectiveness Ratio (ICER) (34).

In addition, the three following ancillary neuroimaging studies were proposed to MAPT participants in selected centers: (i) to explore the effect of interventions on cerebral atrophy rate (total brain and hippocampal volumes), MRI scans were performed at baseline and at 36 months in 9 centers; (ii) to explore the effects of the multidomain intervention on cerebral metabolism, FDGPET scans were performed at baseline, 6 months and 1 year in one site; and (iii) to evaluate amyloïd deposit, florbetapir PET scans were performed once during the three years of the study in 5 PET centers.

\section{Assessment of fatty acids}

Lipids were extracted from red blood cells with a mixture of hexane and isopropanol after acidification. Margaric acid (Sigma) was added as an internal standard. Total lipid extracts were saponified and methylated. Fatty acid methyl esters (FAME) were extracted with pentane and analysed by gas chromatography (GC) using an Agilent Technologies 6890N gas chromatograph with a split injector, a bonded silica capillary column (BPX 70, $60 \mathrm{~m} \times 0.25 \mathrm{~mm} ; 0.25 \mu \mathrm{m}$ film thickness) and a flame ionization detector. Helium was used as a carrier gas, the column temperature program started at $150{ }^{\circ} \mathrm{C}$, increased by $1.3{ }^{\circ} \mathrm{C} / \mathrm{min}$ to $220^{\circ} \mathrm{C}$ and held at $220^{\circ} \mathrm{C}$ for $10 \mathrm{~min}$ (35). Identification of FAME was based on retention times obtained for FAME prepared from fatty acid standards. The area under the curve was determined using ChemStation software (Agilent) and results are expressed as $\%$ of total fatty acids. DHA concentration was calculated using the internal standard and expressed as $\mu \mathrm{g} / \mathrm{g}$ of red blood cells.

Baseline blood samples were assayed by workers blind to the study design and other measurements.

\section{Sample Size}

The sample size required for the MAPT trial was calculated to detect a $0.3 \mathrm{SD}$ difference between the four trial arms (three treatment groups plus placebo group) in the delayed free recall score of the FCRST after 3 years of intervention. To detect a $0.3 \mathrm{SD}$ difference between trial arms, with an alpha risk of $1 \%$ and $80 \%$ power, 201 individuals were required in each group. Anticipating a $30 \%$ dropout over 3 years of intervention, the total sample size required for the study was 1,148 (287 per group). Nevertheless, it was decided to increase the sample size to 1,680 (420 participants per group) in order to account for the high educational level based on data of a previous prevention trial (14).

\section{Study design and follow up (Table 1)}

Follow-up visits are scheduled every 6 months up to 36 months to deliver the supplements, and assess physical condition, the presence of other diseases and corresponding treatments, resource consumption, compliance, tolerability to the omega-3 supplementation, and adherence to the multidomain intervention. Assessments of cognitive and functional status, frailty indicators, and depression are conducted at baseline, six months, and annually at 1,2 and 3 years by research staff blinded to the intervention. All cognitive assessments are performed by hospital practitioners specializing in memory disorders and AD. Fasting blood samples were collected at baseline and 12 months to analyse fatty acid composition in erythrocytes. Additional blood samples are collected and stored at baseline and annually for RNA/DNA extraction. Spontaneously-reported adverse events are recorded to evaluate safety of the intervention.

\section{Interventions}

\section{Omega-3 treatment duration and dose}

The intervention arm will be asked to consume two soft capsules daily as a single dose, containing a total of $400 \mathrm{mg}$ docosahexaenoic acid (DHA), i.e., $800 \mathrm{mg}$ docosahexaenoic acid per day, and a maximum amount of $112.5 \mathrm{mg}$ per capsule of eicosapentenoic acid (EPA) for 3 years, i.e., a maximum amount of $225 \mathrm{mg}$ per day. The placebo arm will be asked to consume two identical soft capsules per day for 3 years. 


\begin{tabular}{|c|c|c|c|c|c|c|c|}
\hline & M0 & M6 & M12 & M18 & M24 & M30 & M36 \\
\hline Demographics & $\mathrm{x}$ & & & & & & \\
\hline Medical history & $x$ & & & & & & \\
\hline Associated pathologies and treatments & $\mathrm{X}$ & $X$ & $X$ & $\mathrm{X}$ & $x$ & $X$ & $X$ \\
\hline Vital signs, clinical examination & $\mathrm{x}$ & $x$ & $\mathrm{X}$ & $\mathrm{X}$ & $x$ & $\mathrm{X}$ & $\mathrm{X}$ \\
\hline ADL & $\mathrm{X}$ & & & & & & \\
\hline MMSE & $\mathrm{x}$ & $X$ & $x$ & & $x$ & & $X$ \\
\hline DSM IV Criteria & $X$ & & & & & & \\
\hline Neuropsychological tests & $x$ & $\mathrm{X}$ & $\mathrm{X}$ & & $x$ & & $x$ \\
\hline - Free and Cued Selective Reminding & & & & & & & \\
\hline - Test Trail Making Test A \& B & & & & & & & \\
\hline - Verbal Fluency & & & & & & & \\
\hline - WAIS-R coding & & & & & & & \\
\hline - Visual Analogue Scale & & & & & & & \\
\hline CDR & $x$ & $x$ & $x$ & & $x$ & & $x$ \\
\hline GDS & $x$ & $X$ & $X$ & & $x$ & & $X$ \\
\hline Functional tests & $x$ & $X$ & $x$ & & $x$ & & $x$ \\
\hline - Fried's criteria & & & & & & & \\
\hline - SPPB & & & & & & & \\
\hline IADL & $x$ & & & & & & $\mathrm{X}$ \\
\hline ADCS-ADL PI & $x$ & $x$ & $x$ & & $X$ & & $x$ \\
\hline Health-economics evaluation & $\mathrm{X}$ & $X$ & $X$ & $\mathrm{X}$ & $X$ & $X$ & $X$ \\
\hline Biology & $x$ & $X$ & $x$ & & $x$ & & $x$ \\
\hline Biological collection & $x$ & & $\mathrm{X}$ & & $x$ & & $\mathrm{X}$ \\
\hline Fatty acid profile & $x$ & & $X$ & & & & \\
\hline Compliance & $x$ & $x$ & $x$ & $\mathrm{X}$ & $X$ & $x$ & $x$ \\
\hline MRI & \multicolumn{2}{|c|}{$\mathrm{X}(\mathrm{M} 0$ or $\mathrm{M} 6)$} & & & & & $x$ \\
\hline FDG-PETscans (monocentric) & $x$ & $x$ & $X$ & & & & \\
\hline AV45- PETscans & $X^{*}$ & $X^{*}$ & $X^{*}$ & $X^{*}$ & $X^{*}$ & & \\
\hline Adverse events & $x$ & $X$ & $X$ & $X$ & $X$ & $X$ & $X$ \\
\hline
\end{tabular}

*AV45 PET scans were proposed either at M0, M6, M12, M18 or M24

\section{Multidomain intervention}

The multidomain intervention $(7,36)$ includes training sessions in the following three areas: nutrition, physical activity, and cognitive training. In addition, individualized preventive outpatient visits exploring possible risk factors for cognitive decline are performed at baseline, 1 year, and 2 years.

Preventive yearly Outpatient Clinics for the MAPT Intervention group have followed the recommendations made by an international task force group (37), which consists of a multidimensional investigation designed to detect hearing or visual disorders, mood disorders, anxiety, malnutrition, walking and balance problems, fear of falling, poor oral and dental health, and vascular risk factors. Good control of vascular risk factors, including management of hypertension, diabetes, and hypercholesterolemia (i.e., known dementia risk factors), is recommended among preventive strategies for dementia $(37,38,39)$.

Training sessions are conducted in small groups (6-8 participants) in twelve 120-minute sessions over the first 2 months (two sessions a week for the first month, and one session a week the second month). Each session includes 60 minutes of cognitive training, 45 minutes of physical training and 15 minutes of nutritional advice.

Starting on the third month, 60-minute sessions each month throughout the 3-year intervention period are conducted to reinforce the key messages of the program and increase compliance. Twenty-minute individual interviews are conducted every 6 months to assess the participant's motivation to change their behavior and elaborate a personalized project. Finally, "booster sessions" (120 minutes) are conducted for each group at 1 year and 2 years. 
Table 2. Baseline data of randomised population $(n=1,680)$

Age, mean (SD), y

$75.3(4.4)$

Sex, number $(\%)$

Female

$1088(64.8)$

Male

$592(35.2)$

Education, number (\%)

No diploma

Primary school certificate

$286(17.4)$

Secondary education, without high-school diploma

$553(33.7)$

High-school diploma (Baccalaureat) or higher

Mini Mental State Examination, mean (SD), /30

$28.1(1.6)$

Clinical Dementia Rating, number $(\%)$

0

$973(58.0)$

0.5

$706(42.0)$

$>0.5$

Free and Cued Selective Reminding Test, mean (SD)

Free recall $(/ 48)$

Total recall $(/ 48)$

$45.2(4.0)$

Delayed free recall $(/ 16)$

$10.6(2.9)$

Delayed total recall (/16)

Trail Making Test, mean (SD), seconds

Part A

$46.6(17.1)$

Part B

$122.7(63.4)$

Verbal fluency, mean (SD), 2 minutes score

Categorical fluency

$25.8(7.5)$

Lexical fluency

$19.7(6.5)$

WAIS-R coding, mean (SD), 90 seconds score

$37.7(10.2)$

Visual Analogue Scale, memory functioning, mean (SD), / 100

$50.0(17.0)$

Visual Analogue Scale, consequences of memory problems in everyday life, mean (SD), / 100

$39.8(23.1)$

Geriatric Depression Scale, mean (SD), / 15

$3.3(2.6)$

ADCS-ADL PI, mean (SD), / 45

$39.7(4.8)$

4-m Gait speed, mean (SD), $\mathrm{m} / \mathrm{s}$

Fried's criteria, number $(\%)$

Involuntary weight loss

Exhaustion

Weakness (handgrip strength)

$378(23.3)$

Slow gait speed

Low physical activity

SPPB, mean (SD), / 12

Blood pressure, mean (SD)

Systolic, $\mathrm{mmHg}$

$141.0(19.7)$

Diastolic, $\mathrm{mmHg}$

Body mass index, mean (SD), $\mathrm{kg} / \mathrm{m}^{2}$

$26.1(4.1)$

\section{Cognitive training}

During the first 2 months, the first 8 training sessions are focused on reasoning and the following 4 sessions on memory. Reasoning training involves teaching strategies for finding the pattern in a letter or word series and identifying the next item in the series. Memory training involves teaching mnemonic strategies (organization, visual imagery, and association) for remembering verbal material (e.g., word lists, sequences of items, text material, or main ideas and details of stories). One of the main objectives of the cognitive sessions is to teach participants how to use these strategies in solving everyday problems (e.g., mnemonic strategies to remember a grocery list, or reasoning strategies to 


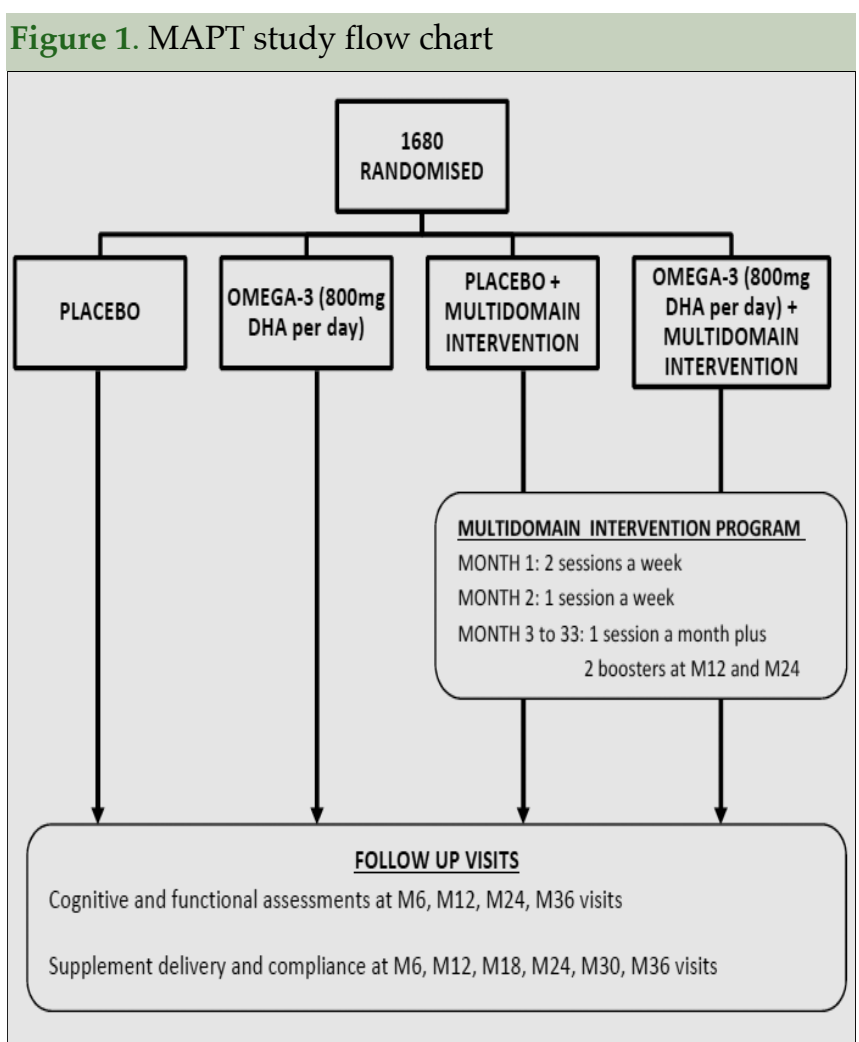

understand the pattern in a bus schedule). The cognitive component of the multidomain program was adapted from ACTIVE and MEMO cognitive intervention (40, 41, 42).

\section{Physical training}

The global aim of the physical intervention is to encourage participants to perform at least 150 minutes of moderately intensive physical activity per week (according to the recommendations of the American College of Sports Medicine) (43). The most frequently recommended type of activity is walking (30 minutes per day). However, participants can choose other forms of exercise to fulfill their five 30-minute sessions per week (e.g., aerobic exercises or strength training activities). The program includes a general advice component and a personalized, home-based physical-activity program, designed with each participant during an individual interview conducted every 6 months (six interviews during the 3 years).

\section{Nutritional advice}

Nutritional advice is based on dietary guidelines established by the French National Nutrition and Health Program for the elderly, which is considered as the official reference in France (44). During the first 2 months eight key guidelines are proposed offering specific recommendations for a healthy diet.

\section{Blinding}

For supplementation, blinding is ensured by the identical appearance (size, color, smell and shape) of the placebo and active capsules.

Training sessions are delivered by qualified trainers. Owing to the nature of the intervention, participants are obviously not blinded with regards to group allocation. At the beginning of the trial and at each subsequent assessment, participants are specifically requested to avoid discussing information regarding the intervention with the research staff conducting the blinded cognitive assessments (in order to limit possible biases in the evaluation), and with other participants (to limit contamination). Participants are also requested to use a diary to record their cognitive, social and physical activities each month.

\section{Adherence}

Unused study supplement capsule is returned at each visit, and compliance with use of the supplement is assessed by tablet count.

Adherence to the program is defined when a subject has attended at least $75 \%$ of the program during the first 2 months (at least 9 of the 12 sessions completed) of the multidomain intervention program.

\section{Preliminary Results}

\section{Baseline Description of the Study population}

Participants were recruited throughout France from May 2008 to February 2011 by 13 centres participating in the study. Details of recruitment strategies were previously published (45). Major sources of recruitment were media advertisement, hospital outpatient clinics and public conferences.

Among the 1680 participants randomized to one of the treatment or placebo arms, spontaneous memory complaint was present in $1664(99.1 \%)$, limitation in one IADL in $188(11.2 \%)$, and slow walking speed in 200 $(11.9 \%)$ at baseline. Two hundred seventy one $(16.2 \%)$ participants reported two of these factors and $51(3.0 \%)$ reported all three factors.

Figure 1 shows the study flowchart and Table 2 shows baseline characteristics for the 1680 participants randomized. Their mean age was $75.3( \pm 4.4)$ years old; there were more women $(64.8 \%)$ than men. A majority of participants $(\mathrm{N}=1272 ; 77.4 \%)$ had received a secondary school education.

\section{Cognitive status}

Mean MMSE at baseline was $28.1( \pm 1.6)$. Fifty eight percent and $42 \%$ of participants had a CDR score equal to 0 and 0.5 , respectively. GDS depression score was 3.3 


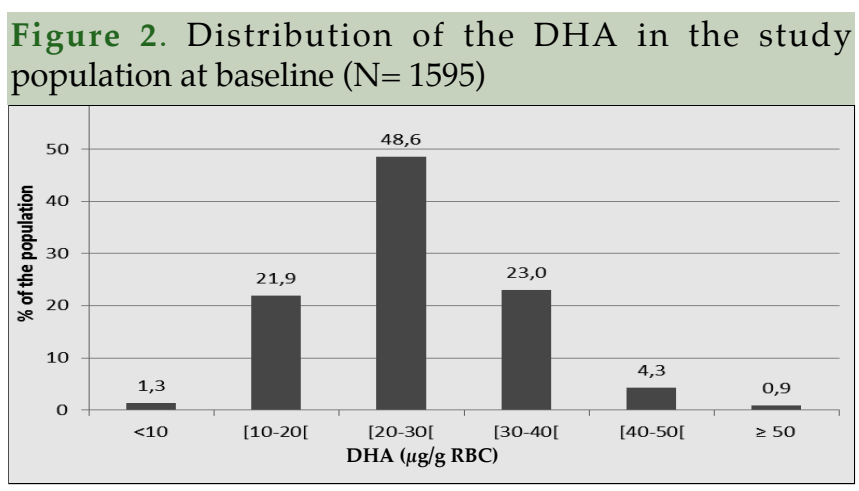

( \pm 2.6$)$. Mean completion times on the TMT were 46.6 $( \pm 17.1)$ seconds (Version A) and $122.7( \pm 63.4)$ seconds (Version B). The mean number of items recalled correctly on the FCSRT test was $45.2( \pm 4.0)$, an amount representing the three free and indexed immediate recalls, and $10.6( \pm 2.9)$ for the delayed free recall. The mean scores on memory function and memory handicap VAS were $50.0( \pm 17.0)$ and $39.8( \pm 23.1)$ respectively. The mean values of other psychometric tests or questionnaires are presented in Table 2.

\section{Autonomy and functional status}

Regarding autonomy and functional capacities, difficulty with at least one item on the IADL was reported for $188(11.2 \%)$ participants. We used the SPPB to assess physical performance. The mean score of SPPB in our sample was equal to $10.6( \pm 1.7)$. One thousand three hundred and two $(78.0 \%)$ participants had a high level physical performance with a score of SPPB equal or higher to 10. Regarding mobility status, mean gait speed was $1.1 \mathrm{~m} / \mathrm{s} \pm 0.3$, with 200 subjects $(11.9 \%)$ reporting a 4$\mathrm{m}$ gait speed lower or equal to $0.8 \mathrm{~m} / \mathrm{s}$.

According to the Fried criteria, $673(42.1 \%)$ participants were considered pre-frail, and $51(3.2 \%)$ frail.

\section{Red Blood Cell (RBC) fatty acids status}

The analysis of RBC fatty acid composition shows a normal distribution with a high variability for DHA content (Figure 2). The mean value is $26.1 \pm 8.1 \mu \mathrm{g} / \mathrm{g}$ with $48.6 \%$ of the population reporting a DHA content between 20 and $30 \mu \mathrm{g}$ DHA / g RBC. The analysis of fatty acid composition (in $\%$ of fatty acid) shows a $4.9 \pm 1.3 \%$ DHA content, in line with common values observed in the general population declaring no treatment or pathologies $(46,47,48)$.

\section{Neuroimaging ancillary studies}

\section{MRI-MAPT ancillary study}

The MRI-MAPT study was conducted in the 7 University Hospital centers (Toulouse, Bordeaux, Montpellier, Limoges, Dijon, Lyon and Nice) and 2 general hospitals (Foix, Tarbes). The first scan was performed on January 21, 2010, and the targeted number of 503 participants was reached on September 21, 2011. 503 participants have undergone baseline MRI (266 subjects accepted to participate at M0 and 237 at M6). A final MRI at the end of the study (month 36) will be performed.

MRI was performed using a standardized protocol provided to the centres. The protocol includes a scan with required sequences (3DT1, T2FLAIR, T2TSE and T2GRE) and optional sequences resting state f-MRI and DTI. Prior to starting MRI acquisition in MAPT participants, centres were requested to test the protocol with a volunteer. Images were then sent to the MRI coordinating centre (CATI and Cogimage), that provided the required certificates of quality. Once purchase was authorized, centres were requested to send the acquired images on a weekly basis. Upon reception by the MRI coordinating center, preliminary quality controls of 3DT1 acquisition were undertaken in order to detect whether a participant needed rescanning.

Preliminary brain segmentation is obtained with the first step of the BrainVISA pipeline. Its aim is to obtain a mask on which to compute the grey/white intensity histogram. Hippocampal volumes were generated using an automated procedure (SACHA Software) (http: / / www.ncbi.nlm.nih.gov.gate2.inist.fr / pubmed/19236922). 30\% of the MAPT participants underwent a brain MRI. This data is presented in Table 3.

\section{FDG-PET ancillary study}

FDG-PET scans were performed at baseline, 6 months and 12 months on 68 participants from Toulouse to assess

\begin{tabular}{|lc|}
\hline Table 3. Baseline characteristics of MAPT-MRI participants (N=503) \\
\hline Mean age at baseline MRI & N (\%) or mean (SD) \\
Female gender (\%) & $74.9(4.0)$ \\
High-school diploma (Baccalaureat) or higher (\%) & $319(63.4)$ \\
Mean MMSE score & $247(49.5)$ \\
Mean total intracranial volume (in $\left.\mathrm{cm}^{3}\right)$ & $28.1(1.6)$ \\
Mean left hippocampal volume (in $\left.\mathrm{cm}^{3}\right)$ & $1375.3(148.4)$ \\
Mean right hippocampal volume (in $\left.\mathrm{cm}^{3}\right)$ & $2.47(0.41)$ \\
Mean Brain Parenchymal fraction $(\%)$ & $2.54(0.43)$ \\
\hline
\end{tabular}


the impact of multidomain intervention on brain metabolism. 34 participants from the multidomain intervention arm and 34 from the control group were recruited for this task. Analysis are in progress.

\section{AV45-PET ancillary study}

Amyloïd PET scans were performed at 5 PET centers (Toulouse, Bordeaux, Montpellier, Limoges and Nice).

The first inclusion was on July 29, 2010, and 271 AV45PET scans were performed during the three years of the study.

Subjects were examined using whole-body hybrid PET-CT scanners, including a PET CT 690 (GE Healthcare), a Discovery RX VCT (General Electric) and three True Point HiRez systems (Siemens Medical Solutions). All scanners operated in 3D detection mode. All PET sinograms were reconstructed with a 3D iterative algorithm, with corrections for randomness, scatter, photon attenuation and decay. This protocol produced images with an isotropic voxel of $2 \times 2 \times 2 \mathrm{~mm} 3$ and a spatial resolution of approximately $5-\mathrm{mm}$ full width at a half maximum at the FOV centre. The acquisition data was processed using the standard package delivered with each acquisition system. All cerebral emission scans began 50 minutes after a mean injection of $4 \mathrm{MBq} / \mathrm{kg}$ weight of $18 \mathrm{~F}-\mathrm{AV} 45$ (florbetapir). For each subject, 10 or 15-minute frames were acquired to ensure movementfree image acquisition.

18F-AV45 (florbetapir) PET images were visually assessed by three independent raters, - specialists trained in molecular imaging and blinded to all clinical and diagnostic information. The raters used a binary scale to classify each scan between 0 (no significant florbetapir cortical retention) and 1 (significant florbetapir cortical retention). Prior to this assessment, the raters underwent a half-day training session on a training set provided by AVID Radiopharmaceuticals Inc.

In addition to the visual readings of scan images, semiautomated quantitative analysis (cortical to cerebellar SUVR) was done using the mean signal of six predefined anatomically relevant cortical regions of interest (frontal, temporal, parietal, precuneus, anterior cingulate, and posterior cingulate) with the whole cerebellum used as a reference region. For this procedure, the $18 \mathrm{~F}-\mathrm{AV} 45$ (florbetapir) PET images were co-registered to the 18FAV45 (florbetapir) template provided by the AVID company and previously published (49). A Quality Control based on semi-quantification process was also provided by AVID Lab. AV45 PET was performed in 271 individuals. Analysis is under progress. Preliminary results show that $38.01 \%(\mathrm{~N}=103)$ had a cortical SUVR > 1.17 , giving indication of significant brain amyloïd deposit. Participants with positive PET scans were of similar age but had lower cognitive functions than those with negative PET scans.

\section{Health Economics of the MAPT Study}

Economic evaluation was performed from the healthcare payer's perspective by recording direct medical and non-medical costs at each visit during the 3year follow up period. Micro-costing is used to value costs. Cost-effectiveness analysis will assess the relationship between costs and outcome (i.e. change of memory functions after a 3-year follow up) between groups of patients.

\section{Follow up visits}

At March 2013, study was completed for 1285 participants and $378(22.5 \%)$ were dropped-out.

\section{Discussion}

Due to the multifactorial aspects of $\mathrm{AD}$, multidomain interventions appear to be an original and potentially effective way to prevent dementia (50). Several target populations must be taken into consideration for preventive trials in Alzheimer's disease. It seems that subjects with Prodromal Alzheimer or Late MCI with brain amyloïd load, reporting a high risk of conversion into dementia, are good candidates for a strong intervention as anti-amyloïd drugs currently tested. A second target population is represented by subjects with early MCI and subjective memory complaints. For example, the A4 trial (Anti-Amyloïd Treatment for Asymptomatic AD Trial) was designed as a secondary prevention trial in clinically normal, $A \beta$ positive individuals who will receive an anti-amyloïd therapy for a 3-year period. (51)

For the current MAPT trial, we targeted older adults with subjective memory complaints, because it is an easy target to be implemented into clinical practice by primary care physicians. We wanted to broaden our population with pre-frail and frail older persons, we were able to include pre-frail older adults but very few frail were included. However, almost $42 \%$ have objective cognitive impairment with CDR 0.5. None have dementia. The omega-3 source used for this trial is not presently commercialized and contains a high dose of DHA (400 $\mathrm{mg}$ per capsule) and a maximum amount of $112.5 \mathrm{mg}$ of EPA per capsule. Our hypothesis is that a high dose of omega-3 during a long period of time is needed to be effective. Indeed, this dose cannot be provided by daily nutritional intake. In our population, the variability of red blood cells DHA content is likely to be linked to natural fish consumption. These results provide a good baseline level of DHA compared to previous studies. For the other fatty acids, nothing appears to be different from common population characteristics.

Some other trials have studied physical exercise, 
nutrition intervention, cognitive exercise, or both $(52,53$, 54,55 ). However the MAPT study is presently the largest trial with such a long- follow up: 3 years for the intervention plus 2 years for the observational follow up (MAPT PLUS extension study). Indeed, it is important to continue the follow up of all MAPT voluntary participants in order to evaluate the long-term effects of preventive strategies. Interestingly, the ACTIVE study group has recently published that this cognitive training had beneficial effects on cognitive abilities and on selfreported IADL ten years later (56). We used interventions that can be easily setup in clinical practice or community health programs and our action appears to hold these challenges. It is important to note that the multidomain intervention was well accepted, with a good compliance during the two first months of intensive program: $77.5 \%$ of participants completed at least nine of the 12 training sessions.

The ancillary imaging studies with brain MRI, FDG PET and Florbetapir PET will give us important data on the potential impact of both interventions on structural imaging and brain metabolism. In MAPT-AV45 trial, 38\% $(\mathrm{N}=103)$ have brain amyloïd deposit. As expected, these individuals have lower cognitive functions than those without amyloïd plaque deposit. It will be interesting to observe if both interventions are able to prevent further amyloïd deposit in those who are negative at baseline. We plan to perform a second PET scan in subjects who are initially negative (three years after the first PET scan).

We are presently looking at APOE4 status in MAPT as well as other genetic markers but they are not yet available. The last patient completed the last visit of the trial in March 2014 and we expect to have the results available at the end of 2014. MAPT trial is the first innovative trial of a large series of European multidomain intervention trials. The MAPT, FINGER and PreDIVA studies joined recently their force in the HATICE program funded by the European Commission (57, 58 ). Other preventive trials (i.e. DO-HEALTH: Vitamin D3 Omega-3 - Home Exercise - Healthy Ageing and Longevity Trial) are on the way with multiple endpoints in aging population.

Abbreviations: FINGER: Finnish Geriatric Intervention Study to Prevent Cognitive Impairment and Disability. PreDIVA: Prevention of Dementia by Intensive Vascular Care. DO-HEALTH: Vitamin D3 - Omega-3 - Home Exercise Healthy Ageing and Longevity Trial. HATICE: Healthy Aging Through Internet Counselling in the Elderly

Funding: This study was supported by grants from the French Ministry of Health (PHRC 2008), and the Institut de Recherche Pierre Fabre (manufacturer of the omega-3 supplement). The promotion of this study was supported by the University Hospital Center of Toulouse. Biological sample collection was supported by Exhonit Therapeutics. AV45-MAPT study was supported by Avid Radiopharmaceuticals Inc.

Conflicts of interest: MAPT study was partially funded by the Institut de Recherche Pierre Fabre, Exhonit Therapeutics and Avid Radiopharmaceuticals Inc.

Ethics: The study protocols have been approved by the French Ethical Committee located in Toulouse (CPP SOOM II) at the date of December 6, 2007 and authorized by French health authority (Ministry of Health) on December 31, 2007.

\section{References}

1. Coley N, Andrieu S, Gardette V, Gillette-Guyonnet S, Sanz C, Vellas B, et al Dementia prevention: methodological explanations for inconsistent results (2008); Epidemiol Rev.30: 35-66.

2. Carrie I, Abellan Van Kan G, Rolland Y, Gillette-Guyonnet S, Vellas B. PUFA for prevention and treatment of dementia? (2009); Curr Pharm Des.15(36): 4173-85.

3. Dacks PA, Shineman DW, Fillit HM. Current evidence for the clinical use of longchain polyunsaturated n-3 fatty acids to prevent age-related cognitive decline and

\section{Appendix. The Members of the MAPT Study Group are}

Principal investigator: Bruno Vellas (Toulouse); Coordination: Sophie Gillette-Guyonnet ; Project leader: Isabelle Carrié ; CRA: Lauréane Brigitte ; Investigators: Catherine Faisant, Françoise Lala, Julien Delrieu ; Psychologists: Emeline Combrouze, Carole Badufle, Audrey Zueras; Methodology, statistical analysis and data management: Sandrine Andrieu, Christelle Cantet, Virginie Gardette , Christophe Morin; Multidomain group: Gabor Abellan Van Kan, Charlotte Dupuy, Yves Rolland (physical and nutritional components), Céline Caillaud, Pierre-Jean Ousset (cognitive component), Françoise Lala (preventive consultation) (Toulouse). The cognitive component was designed in collaboration with Sherry Willis from the University of Seattle, and Sylvie Belleville, Brigitte Gilbert and Francine Fontaine from the University of Montreal.

Co-Investigators in associated centre: Jean-François Dartigues, Isabelle Marcet, Fleur Delva, Alexandra Foubert, Sandrine Cerda (Bordeaux); Marie-Noëlle-Cuffi, Corinne Costes (Castres); Olivier Rouaud, Patrick Manckoundia, Valérie Quipourt, Sophie Marilier, Evelyne Franon (Dijon); Lawrence Bories, Marie-Laure Pader, Marie-France Basset, Bruno Lapoujade, Valérie Faure, Michael Li Yung Tong, Christine Malick-Loiseau, Evelyne Cazaban-Campistron (Foix); Françoise Desclaux, Colette Blatge (Lavaur); Thierry Dantoine, Cécile Laubarie-Mouret, Isabelle Saulnier, Jean-Pierre Clément, Marie-Agnès Picat, Laurence Bernard-Bourzeix, Stéphanie Willebois, Iléana Désormais, Noëlle Cardinaud (Limoges); Marc Bonnefoy, Pierre Livet, Pascale Rebaudet, Claire Gédéon, Catherine Burdet, Flavien Terracol (Lyon), Alain Pesce, Stéphanie Roth, Sylvie Chaillou, Sandrine Louchart (Monaco); Kristelle Sudres, Nicolas Lebrun, Nadège Barro-Belaygues (Montauban); Jacques Touchon, Karim Bennys, Audrey Gabelle, Aurélia Romano, Lynda Touati, Cécilia Marelli, Cécile Pays (Montpellier); Philippe Robert, Franck Le Duff, Claire Gervais, Sébastien Gonfrier (Nice); Yves Gasnier and Serge Bordes, Danièle Begorre, Christian Carpuat, Khaled Khales, Jean-François Lefebvre, Samira Misbah El Idrissi, Pierre Skolil, Jean-Pierre Salles (Tarbes).

MRI group: Carole Dufouil (Bordeaux), Stéphane Lehéricy, Marie Chupin, Jean-François Mangin, Ali Bouhayia (Paris); Michèle Allard (Bordeaux); Frédéric Ricolfi (Dijon); Dominique Dubois (Foix); Marie Paule Bonceour Martel (Limoges); François Cotton (Lyon); Alain Bonafé (Montpellier); Stéphane Chanalet (Nice); Françoise Hugon (Tarbes); Fabrice Bonneville, Christophe Cognard, François Chollet (Toulouse).

PET scans group: Pierre Payoux, Thierry Voisin, Julien Delrieu, Sophie Peiffer, Anne Hitzel, (Toulouse); Michèle Allard (Bordeaux); Michel Zanca (Montpellier); Jacques Monteil (Limoges); Jacques Darcourt (Nice) ;

Medico-economics group: Laurent Molinier, Hélène Derumeaux, Nadège Costa (Toulouse).

Biological sample collection: Christian Vincent, Bertrand Perret, Claire Vinel (Toulouse). 
Alzheimer's disease (2013); J Nutr Health Aging.17(3): 240-51.

4. Barnes DE, Yaffe K. The projected effect of risk factor reduction on Alzheimer's disease prevalence (2011); Lancet Neurol.10(9): 819-28.

5. Coley N, Ousset PJ, Andrieu S, Matheix Fortunet H, Vellas B. Memory complaint to the general practitioner: data from the GuidAge study (2008); J Nutr Health Aging.12(1): 66S-72S

6. Andrieu S, Coley N, Aisen P, Carrillo MC, DeKosky S, Durga J, et al. Methodological issues in primary prevention trials for neurodegenerative dementia (2009); J Alzheimers Dis.16(2): 235-70.

7. Gillette-Guyonnet S, Andrieu S, Dantoine T, Dartigues JF, Touchon J, Vellas B, et al. Commentary on "A roadmap for the prevention of dementia II. Leon Tha Symposium 2008." The Multidomain Alzheimer Preventive Trial (MAPT): a new approach to the prevention of Alzheimer's disease (2009); Alzheimers Dement.5(2): 114-21.

8. American Psychiatric Association. (1994) Diagnostic and statistical manual for Mental Disorders. 4th ed. Washington: American Psychiatric Association.

9. Folstein MF, Folstein SE, McHugh PR. "Mini-mental state". A practical method for grading the cognitive state of patients for the clinician (1975); J Psychiat Res.12(3): 189-98.

10. Katz S, Ford AB, Moskowitz RW, Jackson BA, Jaffe MW. Studies of Illness in the Aged. The Index of Adl: A Standardized Measure of Biological and Psychosocial Function (1963); JAMA.185: 914-9.

11. Peter J, Scheef L, Abdulkadir A, Boecker H, Heneka M, Wagner M, et al. Gray matter atrophy pattern in elderly with subjective memory impairment (2014), Alzheimers Dement.10(1): 99-108.

12. Gifford KA, Liu D, Lu Z, Tripodis Y, Cantwell NG, Pamisano J, et al. The source of cognitive complaints predicts diagnostic conversion differentially among nondemented older adults. (2014 (in press)); Alzheimers Dement.

13. Jessen F, Wolfsgruber S, Wiese B, Bickel H, Mosch E, Kaduszkiewicz H, et al. AD dementia risk in late $\mathrm{MCI}$, in early MCI, and in subjective memory impairmen (2014); Alzheimers Dement.10(1): 76-83.

14. Vellas B, Coley N, Ousset PJ, Berrut G, Dartigues JF, Dubois B, et al. Long-term use of standardised Ginkgo biloba extract for the prevention of Alzheimer' disease (GuidAge): a randomised placebo-controlled trial (2012); Lancet Neurol.11(10): 851-9.

15. Lawton MP, Brody EM. Assessment of older people: self-maintaining and instrumental activities of daily living (1969); Gerontologist.9(3): 179-86.

16. Nourhashemi F, Andrieu S, Gillette-Guyonnet S, Vellas B, Albarede JL, Grandjean $\mathrm{H}$. Instrumental activities of daily living as a potential marker of frailty: a study of 7364 community-dwelling elderly women (the EPIDOS study) (2001); J Geronto A Biol Sci Med Sci.56(7): M448-53

17. Abellan van Kan G, Rolland Y, Bergman H, Morley JE, Kritchevsky SB, Vellas B. The I.A.N.A Task Force on frailty assessment of older people in clinical practice (2008); J Nutr Health Aging.12(1): 29-37.

18. Alfaro-Acha A, Al Snih S, Raji MA, Markides KS, Ottenbacher KJ. Does 8-foo walk time predict cognitive decline in older Mexicans Americans? (2007); J Am Geriatr Soc.55(2): 245-51.

19. Fitzpatrick AL, Buchanan CK, Nahin RL, Dekosky ST, Atkinson HH, Carlson MC, et al. Associations of gait speed and other measures of physical function with cognition in a healthy cohort of elderly persons (2007); J Gerontol A Biol Sci Med Sci.62(11): 1244-51.

20. Van der Linden M, Adam S, Agniel A, Baisset-Mouly F, Bardet F, Coyette F, et al L'évaluation des troubles de la mémoire : présentation de quatre tests de mémoire épisodique (avec leur étalonnage). In: Solal, editor. Marseille2004.

21. Grober E, Buschke H, Crystal H, Bang S, Dresner R. Screening for dementia by memory testing (1988); Neurology.38(6): 900-3.

22. Dubois B, Feldman HH, Jacova C, Cummings JL, Dekosky ST, Barberger-Gateau $\mathrm{P}$, et al. Revising the definition of Alzheimer's disease: a new lexicon (2010), Lancet Neurol.9(11): 1118-27.

23. Vellas B, Andrieu S, Sampaio C, Coley N, Wilcock G, European Task Force G. Endpoints for trials in Alzheimer's disease: a European task force consensus (2008); Lancet Neurol.7(5): 436-50

24. Cardebat D, Doyon B, Puel M, Goulet P, Joanette Y. [Formal and semantic lexical evocation in normal subjects. Performance and dynamics of production as function of sex, age and educational level] (1990); Acta Neurol Belg.90(4): 207-17.

25. Wechsler D. (1981) Wechsler adult intelligence scale-revised. New York: Psychological Corp

26. Reitan R. Validity of the Trail Making Test as an indicator of brain damage. (1958), Percept Mot Skills.8: 271-6.

27. Hughes CP, Berg L, Danziger WL, Coben LA, Martin RL. A new clinical scale for the staging of dementia (1982); Br J Psychiatry.140: 566-72.

28. McNair D, Kahn R. (1983). Self-assessment of cognitive deficits. In: Assessment in clinical psychopharmacology Crook T, Ferris A, Baltus R Ed p. 137-43.

29. Galasko D, Bennett DA, Sano M, Marson D, Kaye J, Edland SD, et al. ADCS Prevention Instrument Project: assessment of instrumental activities of daily living for community-dwelling elderly individuals in dementia prevention clinical trials (2006); Alzheimer Dis Assoc Disord.20(4 Suppl 3): S152-69.

30. Guralnik JM, Ferrucci L, Pieper CF, Leveille SG, Markides KS, Ostir GV, et al. Lower extremity function and subsequent disability: consistency across studies, predictive models, and value of gait speed alone compared with the short physical performance battery (2000); J Gerontol A Biol Sci Med Sci.55(4): M22131 .

31. Fried LP, Ferrucci L, Darer J, Williamson JD, Anderson G. Untangling the concepts of disability, frailty, and comorbidity: implications for improved targeting and care (2004); J Gerontol A Biol Sci Med Sci.59(3): 255-63.

32. Fried LP, Tangen CM, Walston J, Newman AB, Hirsch C, Gottdiener J, et al.
Frailty in older adults: evidence for a phenotype (2001); J Gerontol A Biol Sci Med Sci.56(3): M146-56.

33. Yesavage JA, Brink TL, Rose TL, Lum O, Huang V, Adey M, et al. Development and validation of a geriatric depression screening scale: a preliminary report (1982); J Psychiatr Res.17(1): 37-49.

34. Drummond M, Sculpher M, Torrance G, O'Brien B, Stoddart G. (2005) Methods for the economic evaluation of health care programmes. Third edition ed

35. Legrand P, Schmitt B, Mourot J, Catheline D, Chesneau G, Mireaux M, et al. The consumption of food products from linseed-fed animals maintains erythrocyte omega-3 fatty acids in obese humans (2010); Lipids.45(1): 11-9.

36. Andrieu S, Aboderin I, Baeyens JP, Beard J, Benetos A, Berrut G, et al. IAGC workshop: health promotion program on prevention of late onset dementia (2011); J Nutr Health Aging.15(7): 562-75.

37. Gillette Guyonnet S, Abellan Van Kan G, Andrieu S, Aquino JP, Arbus C, Becq JP et al. Prevention of progression to dementia in the elderly: rationale and proposal for a health-promoting memory consultation (an IANA Task Force) (2008); J Nutr Health Aging.12(8): 520-9.

38. Fratiglioni L, Paillard-Borg S, Winblad B. An active and socially integrated lifestyle in late life might protect against dementia (2004); Lancet Neurol.3(6): 343-53.

39. Kivipelto M, Ngandu T, Laatikainen T, Winblad B, Soininen H, Tuomilehto J. Risk score for the prediction of dementia risk in 20 years among middle aged people: longitudinal, population-based study (2006); Lancet Neurol.5(9): 735-41.

40. Willis SL, Tennstedt SL, Marsiske M, Ball K, Elias J, Koepke KM, et al. Long-term effects of cognitive training on everyday functional outcomes in older adults (2006); JAMA.296(23): 2805-14.

41. Ball K, Berch DB, Helmers KF, Jobe JB, Leveck MD, Marsiske M, et al. Effects of cognitive training interventions with older adults: a randomized controlled trial (2002); JAMA.288(18): 2271-81.

42. Belleville S, Gilbert B, Fontaine F, Gagnon L, Menard E, Gauthier S. Improvement of episodic memory in persons with mild cognitive impairment and healthy older adults: evidence from a cognitive intervention program (2006); Dement Geriatr Cogn Disord.22(5-6): 486-99.

43. Pate RR, Pratt M, Blair SN, Haskell WL, Macera CA, Bouchard C et al Physical activity and public health. A recommendation from the Centers for Disease Control and Prevention and the American College of Sports Medicine (1995); JAMA.273(5): 402-7.

44. Hercberg S, Chat-Yung S, Chaulia M. The French National Nutrition and Health Program: 2001-2006-2010 (2008); Int J Public Health.53(2): 68-77.

45. Carrie I, van Kan GA, Gillette-Guyonnet S, Andrieu S, Dartigues JF, Touchon J, et al. Recruitment strategies for preventive trials. The MAPT study (MultiDomain Alzheimer Preventive Trial) (2012); J Nutr Health Aging.16(4): 355-9.

46. Sanders TA, Ellis FR, Dickerson JW. Studies of vegans: the fatty acid composition of plasma choline phosphoglycerides, erythrocytes, adipose tissue, and breas milk, and some indicators of susceptibility to ischemic heart disease in vegans and omnivore controls (1978); Am J Clin Nutr.31(5): 805-13.

47. Weill P, Schmitt B, Chesneau G, Daniel N, Safraou F, Legrand P. Effects of introducing linseed in livestock diet on blood fatty acid composition of consumers of animal products (2002); Ann Nutr Metab.46(5): 182-91.

48. Sirot V, Dumas C, Desquilbet L, Mariotti F, Legrand P, Catheline D, et al. A restricted cubic spline approach to assess the association between high fat fish intake and red blood cell EPA + DHA content (2012); Nutr Metab Cardiovasc Dis.22(4): 318-26.

49. Joshi AD, Pontecorvo MJ, Clark CM, Carpenter AP, Jennings DL, Sadowsky CH, et al. Performance characteristics of amyloid PET with florbetapir F 18 in patients with alzheimer's disease and cognitively normal subjects (2012); J Nucl Med.53(3): 378-84

50. Schneider N, Yvon C. A review of multidomain interventions to support healthy cognitive ageing (2013); J Nutr Health Aging.17(3): 252-7.

51. Carrillo MC, Brashear HR, Logovinsky V, Ryan JM, Feldman HH, Siemers ER, et al. Can we prevent Alzheimer's disease? Secondary "prevention" trials in Alzheimer's disease (2013); Alzheimers Dement.9(2): 123-31 e1.

52. Bherer L, Erickson KI, Liu-Ambrose T. A Review of the Effects of Physical Activity and Exercise on Cognitive and Brain Functions in Older Adults (2013); J Aging Res.2013: 657508.

53. Wengreen H, Munger RG, Cutler A, Quach A, Bowles A, Corcoran C, et al. Prospective study of Dietary Approaches to Stop Hypertension- and Mediterranean-style dietary patterns and age-related cognitive change: the Cache County Study on Memory, Health and Aging (2013); Am J Clin Nutr.98(5): 126371.

54. Gillette-Guyonnet S, Secher M, Vellas B. Nutrition and neurodegeneration: epidemiological evidence and challenges for future research (2013); $\mathrm{Br} J$ Clin Pharmacol.75(3): 738-55.

55. Ruthirakuhan M, Luedke AC, Tam A, Goel A, Kurji A, Garcia A. Use of physical and intellectual activities and socialization in the management of cognitive decline of aging and in dementia: a review (2012); J Aging Res.2012: 384875.

56. Rebok GW, Ball K, Guey LT, Jones RN, Kim HY, King JW, et al. Ten-Year Effects of the Advanced Cognitive Training for Independent and Vital Elderly Cognitive Training Trial on Cognition and Everyday Functioning in Older Adults (2014); J Am Geriatr Soc.

57. Richard E, Andrieu S, Solomon A, Mangialasche F, Ahtiluoto S, Moll van Charante EP, et al. Methodological challenges in designing dementia prevention trials - the European Dementia Prevention Initiative (EDPI) (2012); J Neurol Sci.322(1-2): 64-70.

58. Dehnel T. The European Dementia Prevention Initiative (2013); Lancet Neurol.12(3): 227-8. 